\title{
An Interactive Agent-Based Learning Environment for Children
}

\author{
Dorin Mircea Popovici, Cédric Buche, Ronan Querrec, Fabrice Harrouet \\ ENIB/CERV, \\ CS 73861, Brest, FRANCE \\ Email: \{popovici,buche,querrec,harrouet\}@enib.fr
}

\begin{abstract}
This paper presents an educational distributed virtual reality-based environment for children called EVE - Environnement Virtuel pour Enfants. EVE is used in elementary schools from different countries as a supplementary tool in teaching children to read. This virtual environment supports cooperation among members of a dispersed team engaged in a shared context. By the mean of their avatars, special cases of agents, children are allowed to interact and to give decisions using cooperative mechanisms. The virtual environment architecture is reactive agents based. The FCMlike dynamic action planning mechanism assures agent's adaptability to its environment changes. It also permits to the pedagogical agent to adapt its behaviour to the child actions. The implementation is based on a client-server architecture, VRML and $\mathrm{C}++$ as languages and ARéVi as graphical rendering API.
\end{abstract}

Keywords: distributed virtual environment, virtual agent, fuzzy cognitive map, intelligent tutoring system.

\section{Motivation}

Learning to read is a difficult but important task for a child. It takes time and supposes a constant effort from its part. As many other school activities, reading involves child's capacities as attention and memory, knowledge (of letters) and know-how as searching and discovering the word's sense in a given context. Small capacity in attention and incapacity to reinvesting in a new task of already known notions are just some examples of obstacles in child's learning process. More, a task failure can generate a fear from child part concerning particular notions, or notions related with the unaccomplished task.

On the other hand, the informal context in which the child learns reading has well evolved in the past three decades. New technologies as multimedia, Internet and Virtual Reality are now parts of our children everyday life. For this reason, it is not surprising that educators grow their interest in distance learning and distributed education, and try to use these technologies in their lessons.

Our paper presents a distributed interactive learning virtual environment (VE), called EVE: Environnement Virtuel pour Enfants (Virtual Environment for Children), which help primary school children learning to read. Based on a story re- construction, it offers children a pleasant approach of learning by means of two games that implements emulation and cooperation.

In the following, after a brief state-of-the-art of educational computer aided environments dedicated to children, we present the EVE project from pedagogical perspective. Next, we will make some considerations from architectural point of view and we give some insights concerning the implementation of our project. Finally, we will make some conclusions based on the experience obtained during the project and we end with perspectives of our work.

\section{Background}

Much of the appeal for applying VR in education is derived from the observations of educational theorists $[1,2]$ who have stressed the value of actualizing learning through making it more real for students. Learning is facilitated through the construction of concepts built from the intuitions that arise out of their direct experience of the environment. The opportunities for young users to visit places and interact with events that distance, time, or safety concerns would normally prohibit, the greater understanding of concepts through the creation of multi-modal metaphors or representations and the ability to scale and manipulate these representations [3, 4], are the major benefits of applying VR in educational environments.

The purposes of these environments are very different. While narrative-based systems as $\mathrm{Oz}[5,6]$, Virtual Theatre [7], NICE [8] or KidsRoom [9] have been mainly developed for entertainment, CityGame [10] study the children' ability to find physically and mentally one's way in the 2D and 3D environments. Round Earth [11] and QuickWorlds projects [12] looks at the issues involved in the use of projection based VR systems with children, specifically in the well known ImmersaDesk [13] or CAVE VR theatres. The effectiveness of multiple representations in enhancing education in elementary school is investigated. Systems as Puppet [14] or GhostWriter [15] goes further and evaluate the use of VE and computer mediated communication technology in the domain of teaching children story writing skills.

As we can see, there are several VR environments that are applied specifically to elementary children learning. Based on a story metaphor, they encourage children to be coconstructors of narration, promoting in this way the deep, connection-building meaning-making activities that define constructivist learning; or suppose the children participation as actors. 
In the environment we present in the following, we have adopted a story-based approach too. Unlike the mentioned projects, we ask the children to discover a proposed story, based on a set of images which are spreaded in a virtual school. Each image is described by a sentence. At its turn, the sentence is constructed using five labels. To discover the story, the children have to reconstruct the sentences and after that to cooperate in order to propose a logical sequence of discovered images. This way, they learn reading by playing.

Based on communication techniques of knowledge and skills, characteristic of intelligent tutoring systems(ITS) [16], EVE is able to adapt the level of the knowledge representation to the children. For this, the agents populating EVE are using internal representation of this knowledge and have the possibility of some reasoning.

\section{The EVE project: general description}

The EVE project has started in 1998. Initially funded by the Fonds Francophone des Inforoutes (Francophone Information Highway Fund), EVE has involved nine partners (Universities, Primary Schools and SMEs) from three countries: France, Morocco and Romania. Since 2000, EVE is used in elementary schools from partner's countries as a supplementary tool in teaching children to read. More, in november 2003 an experience between France and Japan have been realised. Children from the primary school Kroas Saliou (Plouzané, France) have cooperated with children from Ootsukadai elementary school (Yokosuka, Japan).

The target of the project is to implement new cooperative working environments and to initiate new products development such as pedagogical software for primary school children.

\section{Pedagogical aspects}

The primary pedagogical goal is to instill in children the capacity to read and understand a text. If for the reading and phonemes association from six to eighteen month are necessary, for text understanding the child needs a much longer period of time. The latter understanding may become more profound and subtle.

Very often children become able to read without knowing what they are reading. And this represents a problem in all learning processes. How can we resolve a problem in math if we are not able to read and understand its content?

If the children are enthusiasts at the beginning, this state of spirit is calmed down with the time passing because of the long time interval necessary to learn, by the difficulties meet and by the mixture between them. It is strongly necessary that teachers adopt a proper strategy in order to diversify and refresh children interest. Despite the diversity of existed and used educational tools as books, libraries, movies, theatres, educational games, etc., the learning process seems to be to repetitive. And this takes place in the child's life, full of other activities, as sports, playing an instrument, TV, video games, holidays.

The child need of diversity doesn't stop at the school's doors, and our day schools are plugged into this evolution.

\section{Two games}

The EVE application offers children a pleasant approach of learning by means of two games that implements emulation and cooperation.

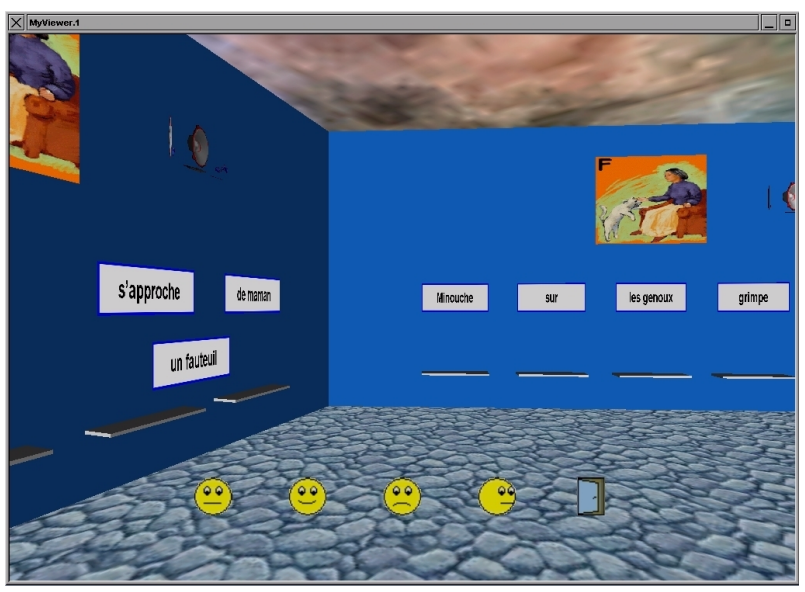

Figure 1: Labels' game.
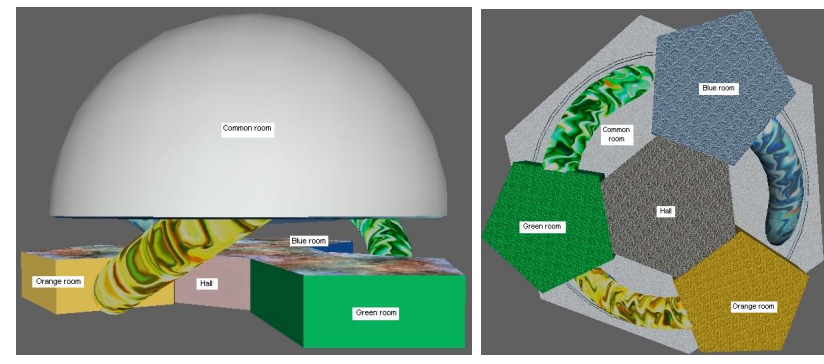

Figure 2: EVE environment (front and bottom-up views).

The labels' game. This first game is a self-training step according to a global learning method of reading; based on the label's game. The target of this first game is to build sentences. A picture illustrates each sentence, in order to suggest to the children the message of the sentence (Fig. 1). The child must discover the sentence using a disordered set of words and moving words to the right places. In case of doubt, the child can hear the sentence by clicking on the appropriate icon. When the sentence is correct the child win the picture. The child must win three pictures before to be able to join the second game. Three children are working concurrently in three different virtual rooms, as orange, green and blue ones (Fig. 2). The order in which the children finish their rooms is the same order they will have the right to express themselves in the next step. More, the first one will be allowed to start the second game. And this motivates them more.

Let's find out the story. Several studies suggest that cooperation between children in virtual environments have a positive effect on learning [11]. It is not very unusual that performances that were expected without results in a child-adult context may appear in a children cooperative context. This is why collaborative learning is one of the most important requirements of our technology. Within a group of children, each child contributes with its knowledge more or less easily acceptable by the others participants.

In the framework of the second game, the spirit of the concurrence make place to the team spirit because of the cooperative context we propose to children. This time the children will meet each other in the common virtual room (Fig. 2). Each child owns a personal avatar. The team of three children must now build the story. For this, they have to place the 

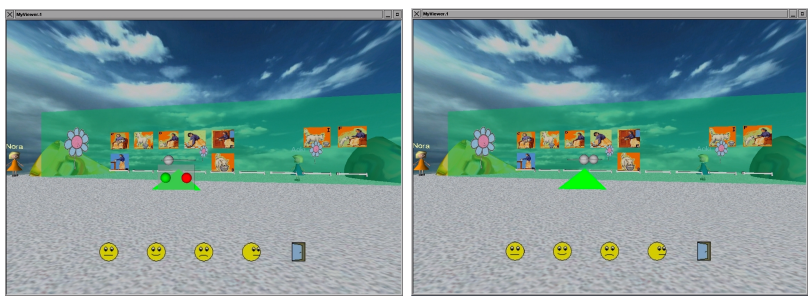

(a) Green team before vote.

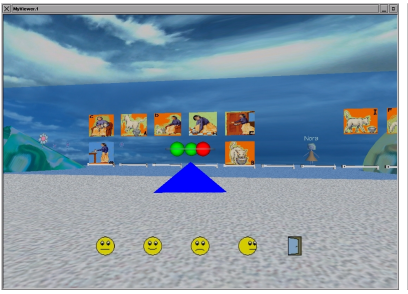

(c) Vote's result.

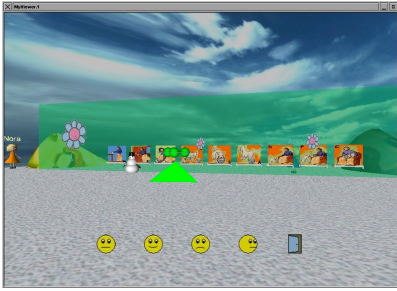

(d) Happy end.

Figure 3: Finding out the story and learning democracy.

previously won pictures in a logic order(Fig. 3). They are allowed to move and propose positions for images in the same order they have finished their rooms. By viewing moving the selected images by the other children, the children are sure that they cooperate and they all have the same goal; even they are in a neighbour room, school or in a foreign country.

A special mechanism is needed in order to avoid conflicts: for example if two children want to move the same picture to two different positions. We have chosen to implement a voting mechanism (Fig. 3):

1. A child moves a picture to a chosen position and requests a vote from the others.

2. The two other children tell him if they agree or not. They are voting (green stands for yes, red for $n o$ ).

3. According to the vote result, which is know only after all the participants have voted, the chosen position for the picture will be accepted or not.

When the team has ended to place all the pictures in an order, if this order is wrong then badly placed pictures are repositioned in original positions and the team must start again to build the story. Otherwise, i.e. the pictures' order is right, an agent (a snowman) comes in the virtual common room and tells the story to the children. This way, the success or the failure of the second game become a team result.

\section{Cultural exchange}

In order to facilitate and so to encourage the multi-cultural exchange EVE supports multi-language working sessions. This way, children can start learning foreign languages, or at least having a first contact with, using the proposed stories. For the moment, in EVE we have stories in French, English, and Japanese (Fig. 4).

\section{Interactivity}

EVE suppose the ability of a child to find physically and mentally ones way in the 3D space. Spatial orientation is an important ability for understanding, interpreting and developing the world in which the children live. Children are given tasks of navigation and orientation inside the environment through

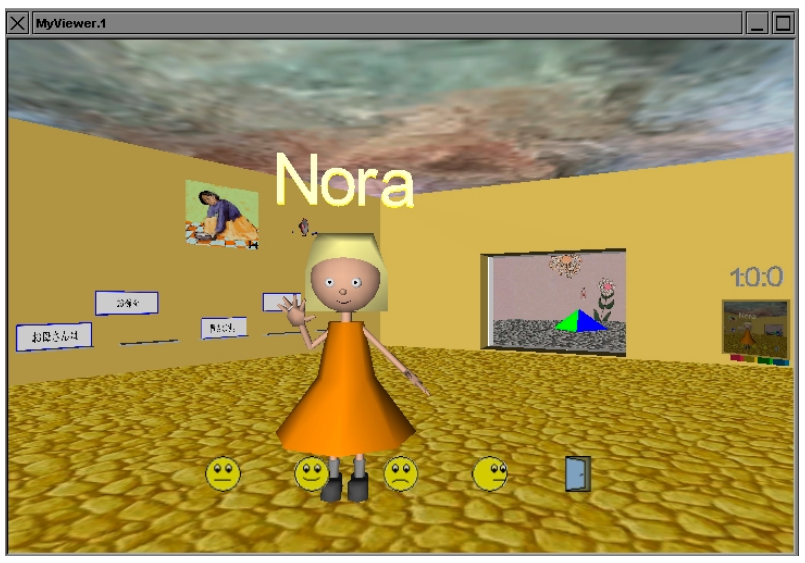

Figure 4: Virtual TV set and scoreboarding.

intersecting paths in order to make them interact. The experienced children with three-dimensional computer games have certainly influenced the behaviour of the subjects.

One important result was that children often focused on the navigation tools rather than on their tasks. More, they love to play 'hide-and-seek' and are eager to see what children in other rooms are doing. The application lets them to perform this task, because emulation concept is the basis of the first game. According to this fact, two mechanisms have been implemented:

1. a scoreboard, which indicates the number of pictures that each child has won, and

2. a virtual TV set, which enables children to view what happens in other rooms. For this, once a TV channel is chosen by the child, the TV screen is connected to the viewer which corresponds with the selected avatar (Fig. 4).

By the mean of avatar's behaviours (Fig. 4) we increase the realism of the virtual representations of children playing in the virtual world and induce a better communication between children. We have chosen to implement behaviours for avatar actions as Walk, Standby, Drag/Drop and used in children communication as Hello!, Good Bye!, Help me!, Thank you!, I agree!, I disagree!.

Simple facial expressions as Neutral, Happy, Unhappy and Laughing are used as a supplement in inter-children communication. These previous lists are not exhaustive and should be completed according to current experiments with teachers and children. The child may select behaviour, which will be broadcasted (gesture and sound) to other children through its personal avatar.

\section{Virtual environment's architecture}

The EVE's environment is populated by virtual entities, briefly called entities, that correspond to objects populating the real world. In our vision, the "meaning" of each entity is its associated object in the real world. As we know, in order to model an object in the real world some essential properties of the object should be used. They will make up the object's meaning from the modeler's point of view. In the virtual environment, for each property of the real object the modeler considers essential will be an attribute of the virtual entity 


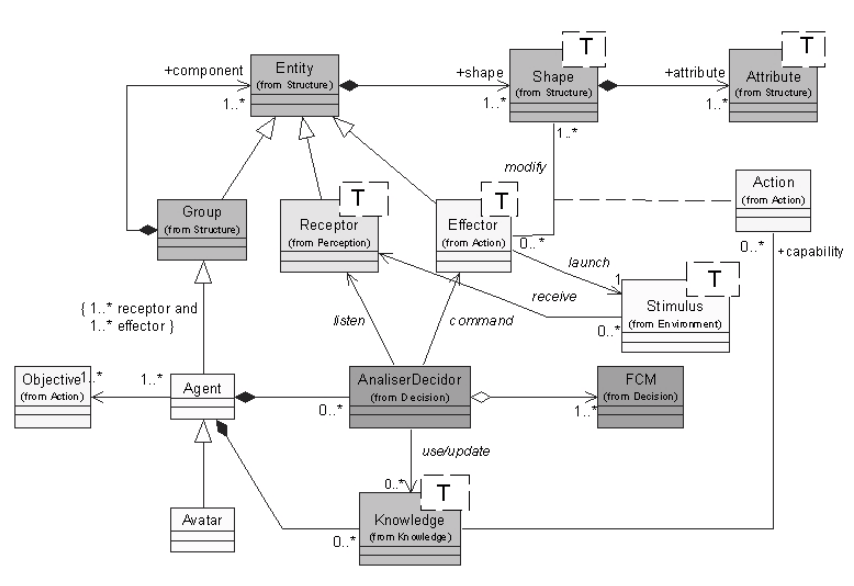

Figure 5: Inside view of the agent's architecture.

with its informational shape so the real object's property is for us the meaning of the attribute associated shape.

In the following, we will consider an entity as the set of all informational shapes which complete its meaning, as in [17] (Fig. 5). By using various criteria, we can structure the set of entities within the virtual environment. For example, the entities can be specialized in receptors and effectors. A receptor is a stimuli detector in an informational space, while an effector realizes shape modifications by means of the entity's actions in the virtual environment. The entities may be grouped in order to produce complex aggregations, themselves entities.

A virtual agent is a complex entity in the virtual environment able to perceive, decide, and react based on its internal structure and tasks. User's avatars are particular cases of virtual agents.

In order to realise the agent's setting in situation into the virtual environment we have followed the immersion, the interaction and the autonomy principles [18]. For this, we were inspired by the human perception mechanisms [19] and we have make use of perception and emission fields of the entities. These are generalisations of medium, nimbus, aura, and awareness notions as they were introduced in [20]. The entities are supposed to populate a multi-dimensional informational space, called virtual environment.

The interactions between agents are of cause-effect type: any change of the agent's state represents a possible cause and may be followed by a stimulus emission. A stimulus contains information regarding the agent state [21]. All the agent's actions are followed by stimuli emissions.

Our virtual agents are autonomous in the measure of their adaptability to their dynamic environment. For this, they have to be able to perceive the environment changes, to decide and to react accordingly (Fig. 6).

During the virtual agent's life, its state is given by the values of its attributes; that are the generators of its structure. The structure's variations are produced by the effectors and are perceived by means of the receptors, under the form of stimuli. These modifications may be triggered by the reception of an external stimulus such as a change in environment followed by emission of internal stimuli.

The receptors from perception module generate perceptions based on these stimuli. The resulting perceptions will be further used by the decision component, which is responsible

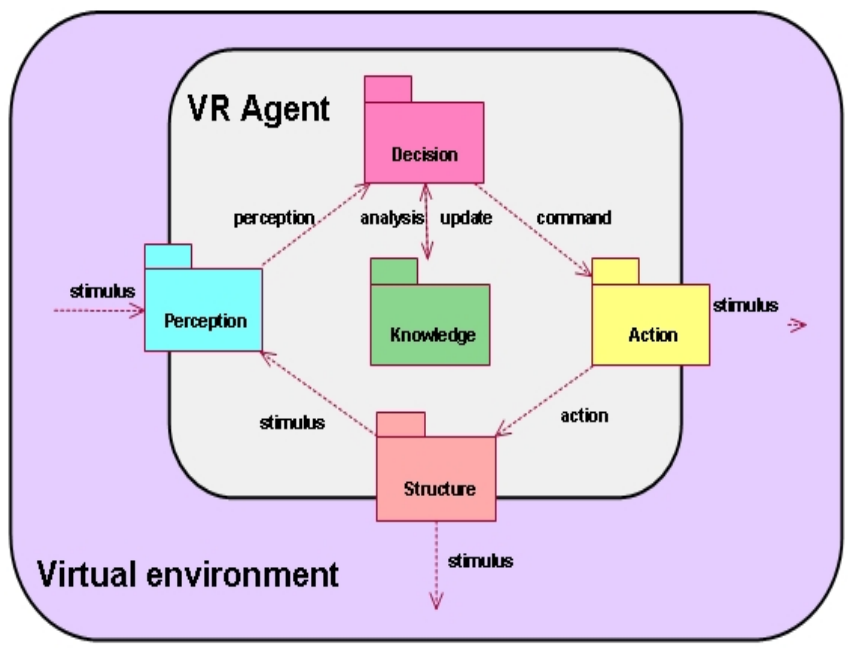

Figure 6: Agent's layers.

for effectors' activation. In their turn, effectors, founded in the action module, may operate structural modifications followed by stimuli emissions, or may trigger themselves specific stimuli. This way, the agent's life cycle is completed.

Information concerning agent's objectives and abilities, as well as its world model, are all stored in its knowledge base. This is the only component which is modifiable not by the means of the agent's effectors but the decider one.

\section{Agent's adaptability to its environment}

The problem that arises concerns the dynamical aspect of the agent's environment. In such an environment, the agent decision upon its actions may become obsolete between the moment the decision is taken and the moment the action is applied. And this is due to the fact that in this time interval agent's action selection mechanism doesn't update the activated action context. In order to eliminate this inconsistency we use a dynamical FCM-like action planning mechanism, based on agent's perception, situated at decision level.

A FCM is an influence graph having as nodes elements of a set of concepts [22]. Concepts may be sensorial (if they express perception values), internal (for knowledge or decisional values), or driving concepts (for actions/objectives values) that the agent possesses [23].

The environment perturbations detected through the agent's receptors will represent imposed values of sensorial concepts. This way, each time the FCM is computed, the agent's behavioural (re)activation is realised.

In our model, not only the agent's objectives are viewed as fuzzy goals [24], but also the action plans. This is because they all have corresponding driving concepts in the decider's fuzzy maps. An action with its corresponding driving concept greater than a specific value is called active; otherwise the action is called inactive.

An action's plan may include different "routes" the agent can follow in order to complete the action. Even if it is unique, the plan's execution can provide different solutions for the corresponding action, depending on the current context. In order to express the action's plan we are using three behavioral patterns, ALL, FOF and SEQ, introduced by the mean of three binary operators, "all", "first of" and "sequence" in [25]. 
With ALL pattern we can express parallel cooperative actions; i.e. we let all the actions to be completed in the same time. By the means of the FOF pattern parallel concurrent actions are expressed. In other words, the first completed action in a FOF plan will determine the plan completion. When an order between the plan's actions is needed, we use the SEQ pattern. This means that the action plan is completed once the last specified action is completed.

\section{EVE's agents}

In EVE we are using two types of agents: agents associated to children - virtual agents - and agents associated to their teachers - pedagogical agents.

\section{Virtual agents}

The main goals of a virtual agent are to Play the game, to Avoid collisions and to Give help. Using our behavioural patterns, these goals may be briefly described as follows (Fig. 7):

$\mathrm{Play}=\mathrm{SEQ}\left(\mathrm{Room}_{1}, \mathrm{Room}_{2}\right)$

$\operatorname{Room}_{1}=\mathrm{SEQ}\left(\right.$ GoInRoom $\left._{1}, \operatorname{ALL}\left(\mathrm{S}_{1}, \mathrm{~S}_{2}, \mathrm{~S}_{3}\right)\right)$, here $\mathrm{S}_{i}$, $i=\overline{1,3}$ represents the reconstruction action of $i$-th sentence in the first room.

$\mathrm{S}_{i}=\mathrm{ALL}\left(\mathrm{Label}_{1}, \ldots, \mathrm{Label}_{5}\right)$

Labe $l_{k}=$ action to place the Label $_{k}$ into the corresponding socket from the corresponding first room walls (Fig. 1).

Room $_{2}=$ SEQ $\left(\right.$ GoInRoom, ALL $\left(\right.$ Img $_{1}, \ldots$, Img $)$, here $\operatorname{Img}_{i}, i=\overline{1,9}$ represents the action's plan to follow in order to place the Image ${ }_{i}$ into an image socket from common room wall.

Img $_{i}=\mathrm{FOF}\left(\mathrm{SEQ}\left(\right.\right.$ Image $_{i}$, AskVote, Vote $)$, Vote) means that the agent will activate the two plans $S E Q$ and Vote but only one will be completed. If it is its turn to propose an image in the common room, then the $S E Q$ wins, otherwise the agent will simply vote.

AvoidCollision, AskVote and Vote are examples of agent's atomic actions.

Give = SEQ (Approach, CurrentLabel) means that the agent will try to Approach to the agent who has send the $\mathrm{Hel}$ pRequest demand. After that, it will activate the CurrentLabel action's plan, i.e. place the current label into the right socket. This time, the action plan components are demanded actions for all the other agents, excepting the agent is asking for help.

This is a typical situation in which the cooperation between agents appears. Once an agent trigger HelpRequest, all the agents in its environment will receive this demand under the form of an external stimulus and will activate their Give help action plan. Due to environment's dynamic (agents positions, access paths, obstacles, etc) only one of these agents will succeed to accomplish this task. The other agents will be informed about this and they will skip the Give help plan and will decide on the next action according to environment context. This way, we have eliminated the obsolete agent's actions due to environment changes during the time interval between agent's action decision and action completion.

\section{The pedagogical agent}

The pedagogical agent is based on the same agent model as virtual one. In addition it has the capability to perceive information in order to construct knowledge based on the four models of traditional ITS:

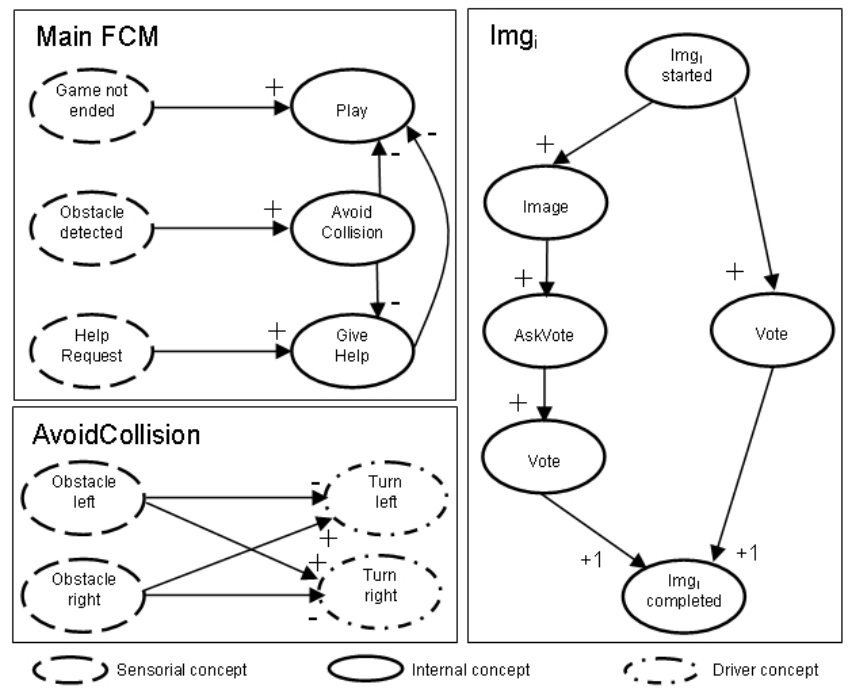

Figure 7: Partial agent's FCMs.

- the expert model is written by the human expert of the domain,

- the rules of the pedagogical model are provided by the pedagogue,

- the student model is dynamically built by the pedagogical agent observing child' actions,

- the interface model is represented by the fact that the child is immerged in the virtual environment

Next we present the expert, student and pedagogical model in EVE.

The expert model. Our expert model is composed of two parts: the declaratory knowledge and the procedural knowledge. In EVE, the first is represented by a simplify set of French grammar rules and the second is constituted by the set of sentences the child has to discover (corrections of the exercise). The point of this model is to detect and to determine the type (semantic or grammatical) of an eventual error made by the child.

The student model. We made the assumption that the pedagogical agent has the knowledge of the FCM structure associated to child's avatar. As we have seen before each time the user activates a concept it broadcast a stimulus. The pedagogical model use this information to update the student model by activating the corresponding concept.

The pedagogical model. The goal is to select pedagogical action(s). We have defined:

1. Say : verbalise the sentence the child has to reconstruct

2. Help : provide the grammar rule the child has to use

3. Show : place a label in its correct socket

4. Ask : ask question about the semantic of the sentence (to evaluate procedural knowledge) or on the grammar (to evaluate declarative knowledge). For example, if the right sentence is "The cat is green." the pedagogical agent could ask "Which color is the cat?" or "Do you think the green is cat is correct?"

5. contradict: evocate an erroneous rule. 


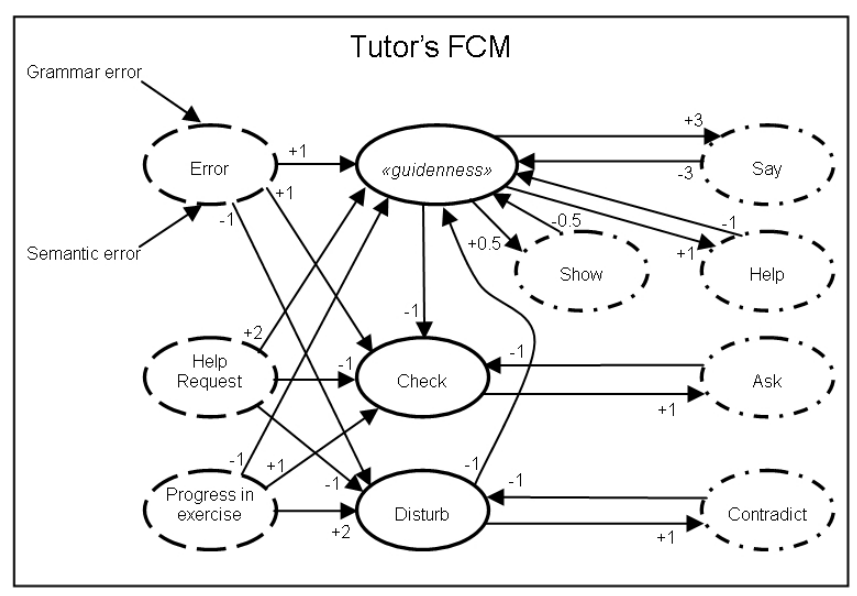

Figure 8: Tutor's FCM

FCM is declarative and explanatory; it can therefore be specified by a non-specialist in computer science (in our case the human teacher). Next, we show an example of such FCM (Fig. 8), but correct one should be provided by an expert in pedagogy. Sensitive concepts are the error (from -the comparison between student model and expert model), the help request and the progress of the exercice (student model). Motor concepts are pedagogical actions. We use different internal concepts as the level of "guideness", the motivation to check or to disturb the learner solution.

Based on the action's plan, we are able to obtain different evaluations of its accomplishment level, either by counting its completed actions or by the means of the objective completed concept value. The greater is this number, the greater may became the objective/action priority from the agent's perspective.

The action's corresponding FCM convergence to a fixedpoint attractor assure the action's accomplishment or failure, depending on the value of completed concept at this point.

If there are some concurrent actions with similar effects, then they are let to cooperate, otherwise the action with lower priority is made inactive. After the currently active action will be accomplished, the rest of the actions will be re-evaluated and activated accordingly.

We have to remind that the associated effectors control the values propagation through a fuzzy-like plan. This makes them different than but as simple as classical FCMs.

\section{Implementation}

\section{EVE distribution}

The EVE distribution is client-server based.

The main function of the EVE server is to organise and manage the users' connection into the set of existing stories. Once the users have chosen a story, on the server site all the messages are broadcasted in the same order to all clients who are playing in the same story.

On the client side, the user's immersion into the EVE enviroment is realised by the means of an ARéVi API-based application [26]. This interface allows the user to carry out complex tasks by mixing the implemented behaviors. More, the users movements are restricted to EVE environment and depend on his/her attributes (height, step size, etc).
A dead-reckoning pseudo-mechanism is also used. In order to avoid the network overloading, we filter the input events generated by actions on the input devices.

At the startup, the client loads the EVE environment (as VRML and multimedia resources). When the user logs in, the client adds all active avatars to the virtual world. Then it attaches the interaction-navigation metaphor. Finally, it updates the avatars state depending on their behavior, in the local replica of the virtual world.

\section{The environment}

Virtual environment and its components have been designed with Virtual Reality Modeling Language (VRML) ISO standard, and all have a semantic meaning (room, door, button, teleport device, etc). This way, an agent in EVE is able to navigate within the environment without having a pre-recorded map of the environment.

\section{The agents}

The virtual agents were designed on an approximate-body approach [27] which provides frequently position and orientation information to remote hosts, taking into account a minimal set of joint points. They were implemented based on the architecture presented in figure 5 as ARéVi C++ objects. Their geometrical structure components were implemented in VRML.

The agent's atomic actions were considered as imperative methods and forms the agent's capabilities. They are used in the actions' plan realization according to figure 7 .

More, using virtual agents we are able to ensure the persistence of the game. Let us suppose that there is a child who wants to quit the game before its end. In this case, without a virtual agent who takes the child's place, the game will be blocked. But using a virtual agent, the child can quit the game without affecting its flow, its role being switched to the corresponding virtual agent.

\section{Conclusions}

In this paper we have presented a virtual environment specially dedicated to pedagogical purposes. The application is successfully in use as a complement of traditional learning exercises in primary schools from France, Morocco, Romania and Japan. Such exercises already exist using scissors and paper. But the difference here is twofold:

1. children get an immediate validation of their works, for example an animal appears when the sentence is correct;

2. children don't realize that they are working; in fact they feel as if they were playing together.

Experiments have pointed out that games increase children motivation, and new technologies such as virtual reality increase children autonomy. More, they are able to take over the virtual world and to move into the virtual space. For example, after a short time of practice, children have transposed into the virtual world one of their real game: hide-and-seek. The cooperative work demonstrates that children in a multicultural framework are able to work together if they comply with common rules.

The second game (Fig. 3) is meant to text understanding and logics. Generally children have difficulties to read and to correct themselves. They often make the same mistakes. Here, it is impossible because they have to change something 
if they are wrong; they have to build a new reasoning. This point out that our application contributes to increase the ability of children to reason by themselves.

On a pedagogical point of view, planned for the primary courses, EVE is now adaptable for older children. The teacher may pass from a text comprehension to grammar or syntax studies. More, new applications should be developed in other domains of interest such as: foreign languages, geometry, algebra, and not only. More, as stories are shared between classrooms or schools, it increases the quantity of exercises teachers can propose to children.

On a technical point of view, current experiments will help us to design and implement new avatar behaviours according to end users' needs. We are also working on a more sophisticated mechanism, which is the integration of streaming audio and video into the virtual world. This should increase the performance of children cooperative work.

Emotional and social aspects of agent's behaviour as well as the validation of critical action's plans (like cyclic ones), are some of our priorities. The integration of the pedagogical agent in EVE is in progress. For this, we cooperate with experts in pedagogy in order to obtain a correct behavior implemented using FCM. So, the pedagogical agent, which actually assist children, will participate into the voting mechanism, or will generate mistakes in order to supplementary test the children, as a higher level of children's pedagogical evaluation.

To conclude, we believe that Virtual Reality is likely to enable the development of new products that will help children and teachers in their tasks of learning and teaching through friendly interfaces. Finally, cooperative experiments pointed out that such cooperative work introduces a kind of subliminal target, which is: training children in learning democracy.

\section{References}

[1] J. Piaget. The Child's Conception of the World. NY: Harcourt-Brace, New York, 1929.

[2] J. Bruner. Actual Minds, Possible Worlds. Harvard University Press, Cambridge, MA, 1986.

[3] W. Winn. A conceptual basis for educationam applications of virtual reality. Technical Report TR 93-9, 1993.

[4] C. Youngblut. Educational uses of virtual reality technology. In Technical Report IDA Document D-2128, Institute for Defense Analyses, Alexandria, VA, 1998.

[5] J. Bates. Virtual reality, art and entertainment. PRESENCE: Teleoperators and Virtual Environments, 1(1):133-138, 1992.

[6] M. Mateas. An oz-centric view of interactive drama and believable agents. In Technical Report CMU-CS97-156, 1997.

[7] B. Hayes-Roth and R. vanGent. Story-making with improvisional puppets. In Proceedings of First International Conference on Autonomous Agents, pages 1-7, 1997.

[8] M. Roussos, A. Johnson, T. Moher, J. Leigh, C. Vasilakis, and C. Barnes. Learning and building together in an immersive virtual world. Presence, 8(3):247-263, 1999.
[9] A. Bobick, S. Intille, J. Davis, F. Baird, C. Pinhanez, L. Campbell, Y. Ivanov, A. Schutte, and A. Wilson. The kidsroom - a perceptually-based interactive and immersive story environment. Presence: Teleoperators and Virtual Environments, 8(4):367-391, 2000.

[10] S. Volbracht, G. Domik, D. Backe-Neuwaldand, and H.D. Rinkens. The "CiTy GAME" an example of a virtual environment for teaching spatial orientation. Journal of Universal Computer Science, 4(4):461-465, 1998.

[11] A. Johnson, T. Moher, S. Ohlssoon, and M. Gillingham. The round earth project - collaborative vr for conceptual learning. IEEE Computer Graphics and Application, Virtual Reality, 19(6), 1999.

[12] A. Johnson, T. Moher, J. Leigh, and Y. Lin. Quickworlds: Teacher driven vr worlds in an elementary school curriculum. In Proceedings of SIGGRAPH 2000 - Educators Program, New Orleans LA, 2000.

[13] A. Johnson, T. Moher, Y.J. Cho, Y.J. Lin, D. Haas, and J. Kim. Augmenting elementary school education with vr. IEEE Computer Graphics and Applications, March/April:6-9, 2002.

[14] P. Marshall, Y. Rogers, and M. Scaife. Puppet: a virtual environment for children to act and direct interactive narratives. In 2nd International Workshop on Narrative and Interactive Learning Environments, Edinburgh, 2002.

[15] J. Robertson. Computer games - for better or for worse? i3 magazine, 12:32-34, 2002.

[16] E. Wenger. Artificial Intelligence and Tutoring Systems. Morgan Kaufmann, 1987.

[17] D.M.Popovici, L.D.Serbanati, and F.Harrouet. The virtual environment - another approach. In WSCG'2003 Posters Proceedings, pages 109-112, Plzen, Czech Republic, 2003.

[18] J. Tisseau and F. Harrouet. Autonomie des entités virtuelles. In Le traité de la réalité virtuelle, volume 2, pages 85-124, Les Presses de l'Ecole des Mines, Paris, 2003.

[19] P. Herrero and A. De Antonio. A human based perception model for cooperative intelligent virtual agents. In (CoopIS' 02)Tenth International Conference on Cooperative Information Systems, pages 195-212, Irvine, California, USA, 2002.

[20] S. D. Benford and L. E. Fahln. A spatial model of interaction in large virtual environments. In Proc. EC$S C W^{\prime} 93$, pages 109-124, Milano, Italy, 1993. Kluwer Academic.

[21] D.M.Popovici, L.D.Serbanati, and J.P.Gerval. Agentbased modeling of virtual environments. In Proceedings of VRIC 2003, pages 149-158, Laval, France, 2003.

[22] B. Kosko. Fuzzy Engineering. Prentice Hall Inc., 1997.

[23] M. Parenthoen, P. Reignier, and J. Tisseau. Put fuzzy cognitive maps to work in virtual worlds. In FuzzIEEE01, Melbourne, Australia, 2001.

[24] M. S. El-Nasr, J. Yen, and T. Ioerger. Flame - a fuzzy logic adaptive model of emotions. Autonomous Agents and Multi-Agents Systems, 3:219-257, 2000. 
[25] D.M.Popovici, J.P.Gerval, P.Chevaillier, J.Tisseau, L.D.Serbanati, and P.Gueguen. Educative distributed virtual environments for children (to appear). JDET, 2(4):18-40, 2004.

[26] P.Reignier, F.Harrouet, S.Morvan, J.Tisseau, and T.Duval. Arévi : a virtual reality multiagent platform. In Proceedings VW'98, Paris, july 1-3, pages 229-240. Springer Verlag, LNAI1434, 1998.

[27] D. Thalmann. Challenges for the research in virtual humans. In Workshop Achieving Human-like behavior in interactive animated agent, Barcelona, Spain, 2000. 